\title{
Disease Types and Processing Measures in Perennial Work Stop- page Tunnels
}

\author{
Qi He ${ }^{\mathrm{a}^{*}}$, Kaixi Xue ${ }^{\mathrm{b}}$, Hengjun $\mathrm{Hou}^{\mathrm{c}}$, Jiashuai Chen ${ }^{\mathrm{d}}$, Weiqing Feng ${ }^{\mathrm{e}}$, Bobo Bai ${ }^{\dagger}$, \\ Xinwu Zhao ${ }^{g}$, Kailiang $\mathrm{Xu}^{\mathrm{h}}$
}

College of Civil and Architecture Engineering, East China Institute of Technology, Nanchang, Jiangxi, China.

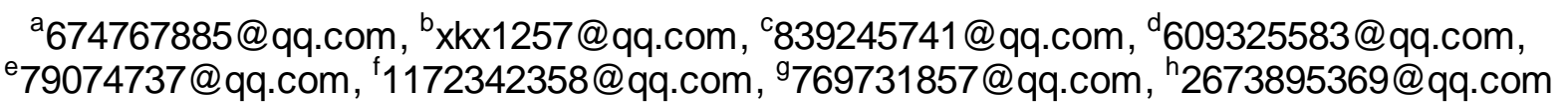

Keywords: Expressway continual construction projects; Perennial work stoppage tunnels; Disease causes; Treatment measures; Treatment suggestions

Abstract: This paper presents an example of the Pinghong(Pingxiang to Hongkoujie) expressway in Jiangxi province continual construction project, focusing on identification of disease types, analysis of disease causes, as well as to the measures and suggestions for disease treatments. It can provide references for future tunnel design, construction and management.

\section{Introduction}

A fast, convenient and efficient transportation condition has brought many benefits for the development of the national economy and the improvement of the residents' living environment. People's awareness of the importance of expressway increases strongly. Expressway construction investment scale is huge, underfunded investment will cause the work cessation. Without any effective protection, the perennial work stoppage expressway project, diseases of the built project are more than the new one. Structures are in the unreasonable interface after long-time shutdown. Before the resumption of expressway construction, the partly lost quality check file data gives the quality evaluation of built projects troubles so much as the quality of concealed work. Based on the complex characteristics of continual construction projects, identification of disease types provides the basis for the evaluation of the reliability, the analysis of the reliability and durability for the continual construction projects and the completion of projects.

A recent domestic study, some scholars have studied on the disease types and causes of expressway bridges of continual construction projects, putting forward the corresponding measures [1]. At present, domestic and overseas scholars have researched in disease types, the causes of disease, disease treatment measures and suggestions of new and built tunnels. But, it is still in the blank for perennial work stoppage ones.

This paper will systematically research in three aspects: the disease type, the cause of the disease, the treatment measure and suggestion for diseases of perennial work stoppage tunnels.

\section{THE DISEASE TYPE AND CAUSE ANALYSIS}

\section{Temporary Slop Collapse of the Tunnel Portal and Its Cause}

There are some problems of "difficulties into the entrance and the exit" in the tunnel engineering. But, the main problem, the temporary slope stability relates to the success or failure of the whole tunnel engineering [2]. There are three separation-type tunnels: Changping tunnel, Mingshan tunnel, Jiaoyuan tunnel and one multi-arch tunnel: Hongkoujie tunnel on Pinghong(Pingxiang-Hongkoujie) expressway. The right line entrance of Jiaoyuan tunnel is designed for the side wall style, and the second stair of temporary slope has been excavated. But, it didn't be taken the slope supporting measures. So, the temporary slope of the tunnel portal has collapsed. The entrance of Hongkoujie tunnel is designed for bamboo-cutting style, and the 
second stair of temporary slope has been excavated. But, it also didn't be taken the slope supporting measures. So, the temporary slope of the tunnel portal has collapsed.

The top of the mountain where tunnels passed through, covers with the brae residual sediments. Their structure is loose. It is the characteristics of good water permeability, poor mechanical performance, low shear strength and easy softening under the influence of water. And the bedrock stratification inclination coordinates with the slope inclination, the downhill joints, and the angle is less than the comprehensive governance angle. Slope excavation may lead to collapse and landslide along the bedding. With the tunnel portal slope excavation, the slope occurs within stress redistribution and stress concentration at the top of slopes. Due to the low tensile strength of rock mass, rock mass damage extremely easy in the role of tensile stress, producing a crack. With the formation of tension crack, the strength of rock mass decreases rapidly. The discontinuity surface occurring, changes the boundary condition of the slope rock mass, which inevitably causes stress distribution further adjustments in the slope. Within new slope deformation space by tunnel excavation, the internal stress distribution in the slope changes. It will certainly cause slope deformation and failure. When stress redistribution, the slope can maintain balance, slope deformation shows as surface movement and subsidence. The destruction of the slope appears on cracking at the top of a slope and shear failure at the toe of the slope [3]. During rainy season, the slope without taking any supporting measures and imperfect tunnel section water gutter damaged, causing drainage function is not smooth. Rain infiltrate into the slope through fractures, exacerbating the deformation and failure of rock mass slope, as the front of the anti-sliding segment excavated, which reduces the slope stability, the slope is not able to maintain balance, the deformation and failure is characterized by continuous sliding, and eventually resulting in the whole slope collapse.

The Tunnel Primary Retaining and Protecting Steel Frames and Crossings Corroding and Its Cause

The left line entrance of Mingshan tunnel ZK6 + 476 to ZK6 + 611 sections, the right line entrance YK6 + 490 to YK6 + 533 sections, the left line exit ZK8 + 456 to ZK8 + 382 sections, ZK8 + 348 to ZK8 + 344 sections and the left line exit YK8 + 470 to YK8 + 414 sections, Steel Frames and Steel Crossings have Corroded.

The left line entrance of Jiaoyuan tunnel ZK9 + 330 to ZK9 + 400 sections, ZK9 + 523 to ZK9 + 632 sections, ZK9 + 653 to ZK9 + 659 sections, Steel Frames and Steel Crossings have Corroded.

The left line exit of Changping tunnel ZK10 + 958 to ZK10 + 579 sections, the right line exit YK10 + 945 to YK10 + 945 sections, YK10 + 775 to YK10 + 760 sections, Steel Frames and Steel Crossings have Corroded.

New poured concrete with high alkaline $(\mathrm{PH}>12.5)$, can guarantee the stability of the passive film and make the steel bar corrosion will not occur [4]. Most of tunnels after excavation have been carried on the primary retaining and protecting structures. But, only a small part of tunnels are in a state of bare rock without the primary retaining and protecting structures, due to perennial work stoppage, not been carried on the secondary lining in time and taken effective protective measures, cracks in tunnel primary supporting. And, the concrete has been carbonized by air and water. Concrete carbonization can make the loss of reinforced concrete protective effect, causing steel frames and rebar surface depassivation effect. When the oxygen and water exist in the surface of steel, rebar corrosion occurs. Steel frames, the depassivated rebar as the anode for oxygen reduction reaction to provide electronic. Steel frames, rebars of intact passive membrane form cathode, for oxygen reduction reaction. Cathodic reaction products through the concrete pore solution transportation near the anode, and the anode reaction products further reaction to generate hydrogen ferrous oxide and iron hydroxide [5,6].

\section{Cracks of Tunnel Gunite Concrete and Its Cause}

Crack width of the left line entrance of Mingshan tunnel is range from $0.12 \mathrm{~mm}$ to $0.89 \mathrm{~mm}$, maximum crack width of $0.89 \mathrm{~mm}$ in the ZK6 +497.61 section on the left wall, and cracks are given priority to with vertical fracture, no obvious cracks fault. Crack width of the right line entrance of Mingshan tunnel is range 
from $0.12 \mathrm{~mm}$ to $0.30 \mathrm{~mm}$, maximum crack width of $0.30 \mathrm{~mm}$ in the YK6 + 505.596 section on the right side of the wall, with vertical cracks. Crack width of the left line exit of Mingshan tunnel range from 0.12 $\mathrm{mm}$ to $0.98 \mathrm{~mm}$, maximum crack width of $0.98 \mathrm{~mm}$, in the ZK8 + 418.41section on the left wall, and cracks are given priority to with vertical fracture, no obvious cracks fault. Crack width of the right line exit of Mingshan tunnel is range from $0.20 \mathrm{~mm}$ to $1.4 \mathrm{~mm}$, maximum crack width of $1.4 \mathrm{~mm}$ in the YK $8+$ 469.09 section on the left wall, and cracks are given priority to with vertical cracks. Crack width of the left line entrance of Jiaoyuan tunnel interval is range from $0.12 \mathrm{~mm}$ to $0.44 \mathrm{~mm}$, maximum crack width of 0.44 $\mathrm{mm}$ in the ZK9 + 424.34 section on the left wall, and cracks are given priority to with vertical fracture, no obvious cracks fault. Crack width of the right line entrance of Jiaoyuan tunnel is range from0.20 mm to 2.4 $\mathrm{mm}$, maximum crack width of $2.4 \mathrm{~mm}$ in the YK9 +352.06 section on the left wall, and cracks are given priority to with vertical cracks. Crack width of the left line exit of Changping tunnel is range from $0.18 \mathrm{~mm}$ to $0.50 \mathrm{~mm}$, and cracks are given priority to with vertical and horizontal cracks, no obvious fault cracks. Crack width of the right line exit of Changping tunnel is range from $0.12 \mathrm{~mm}$ to $0.54 \mathrm{~mm}$, and cracks are given priority to with toroidal and transverse cracks, more the ring fracture on the left side, more the transverse crack on the right side, no obvious fault cracks.

Tunnel gunite concrete cracks are given priority to with vertical cracks, due to the disorder of perennial work stoppage, failed to timely casting secondary lining, and failure to take effective measures, causing the gunite concrete of the tunnel lack of maintenance, prolonged exposure to air, has experienced many times of dry-wet alternate cycle, evaporation of the gunite concrete. The gradual development of its excellent evaporation drying process lead to vertical drying shrinkage cracks of the gunite concrete.

Tunnel surrounding rock geological changes, the longitudinal uneven load, settlement joint disposal is underserved, deformation of surrounding rock unloading with the junction of integral rock and bad geological belts exceed the strength of the gunite concrete generating ring cracks.

Thickness of primary retaining and protecting structures in tunnels did not conform to design requirements, and tunnel excavation settlement deformation of surrounding rock exceeded the strength of the gunite concrete which generated transverse cracks.

\section{The Insufficient Thickness of Gunite Concrete and Its Cause}

Thickness of constructed tunnel section, the measured average thickness is less than $40 \%$ of gunite lining design, difference between $0 \mathrm{~cm}$ and $2 \mathrm{~cm}$.

The effect of smooth blasting of the tunnel surrounding rock excavation is poor, which leaded to the tunnel surrounding rock surface concave and convex drama, or gunite nozzle jet direction did not remain perpendicular to the rock surface, which leaded to the insufficient thickness of gunite concrete.

\section{Tunnel Lining Cavity and Its Cause}

The vault, hance and side wall of part sections of the tunnel lack of necessary compactness, had cavities.

The tunnel passes through in complex geological conditions, the upper is quaternary residual materials, slope bottom bedrock structure developing sharply, crushing of rock mass, rock tunnel smooth blasting effect is bad, some companies want to save the cost, setting stones or other foreign material to replace concrete filling space through steel fabric behind shotcrete layer, causing unreasonable compactness between surrounding rock and initial retaining and protecting structures, even forming a big empty [7]. Before surrounding rock shotcrete working, failure to clean up the collapse of earth-rock on tunnel excavation, deformation not be effectively controlled, gunite concrete appears empty after the test.

\section{Percolating Water of Tunnel Lining and Its Cause}

Construction joint in ring and ring cracks appears water seepage trace at the left line entrance of Mingshan tunnel, local water burst. The vertical cracks appear local water seepage trace at the line right entrance of Mingshan tunnel. The vertical cracks appear local water seepage trace at the right line exit of Mingshan tunnel. Leakage flow of circular construction joint and annular crack are not obvious at the left line entrance of Jiaoyuan tunnel, only form of dry and wet stained stains. Leakage in vertical fracture is not 
obvious at the right line entrance of Jiaoyuan tunnel, only the manifestation of a dry wet stained and stains. Leakage flow in toroidal construction joint and annular crack are not obvious at the left line exit of Changping tunnel, only form of dry and wet stained stains. Leakage is not obvious at the right line exit of Changping tunnel, toroidal construction joints, circular cracks and the junction between arch feet and sidewall, only the manifestation of a dry wet stained and stains.

Water-stop belts and waterproof layers buried in the tunnel primary lining construction joints have problems of lap joint, not easy to form a closed circle of waterproof. In addition, damaged water-stop belts, the location set is not straight and not vibrating compaction of concrete around water-stop, especially in the underside of the arch of osteoporosis and accumulated quite a lot of air bubbles to form water access [8]. The tunnel primary lining exposed to the air for a long time, has experienced many times of dry-wet alternate cycle, evaporation of gunite concrete, a vertical drying shrinkage cracks. Tunnel surrounding rock geological changes, the longitudinal uneven load and settlement joint disposal is undeserved, ring cracks in junction of bad geological belts and intact rock. Precipitation rain period brings the weathering fissure water and karst water, weathering fissure water and karst water leakaging through vertical and circular cracks.

\section{Karst Cave Collapse of Tunnel Hance and Its Cause}

The right line entrance of Mingshan tunnel, the arch on the right side of YK6 + 553 to YK6 + 553 sections appeared a diameter of 2 meters grown cave, collapsed at the hance.

Mingshan tunnel passes through the widespread karst region, and in the tectonic fault zone development area, developed karst caves, because of treatment of karst caves not in time before. And secondary lining didn't cast in time for perennial work stoppage, after the tunnel primary retaining and protecting structures, causing the development of karst cave collapse and I shape cross section steel hance flunking.

\section{Insufficient Headspace of Tunnel Outline and Its Cause}

The local parts paragraphs of tunnel primary retaining and protecting structures appeared into the second lining no more than $50 \%$ of this section.

After tunnel excavation, surrounding rock stress released, vault and soft base, failed to timely casting the secondary lining after the completion of the tunnel primary retaining and protecting structures, strong arch pressure. Settlement of surrounding rock and convergence had not been effectively controlled after perennial work stoppage, the primary retaining and protecting structures was not enough to resist outside pressure, the tunnel primary support invaded the second lining.

\section{TREATMENT MEASURES AND SUGGESTIONS}

\section{Treatment Measures and Suggestions for Temporary Slope Collapse of Tunnel Portal}

(1) the tunnel primary supporting is carried on corresponding support within the scope of the radial grouting, with the I-shaped cross section NO.16 beam as a support frame, the upper points within the original hole, the initial supporting of the I-shaped steel, lower points to fall on the stable foundation, to ensure that the primary support system does not move even not collapse in the process of grouting or digging holes.

(2) Inside the hole on the upper tunnel portal geotechnical radial grouting reinforcement, firming excavation contour line range of rock mass, preventing the upper rock mass sliding in the process of excavation and lateral pressure, avoid to have a harmful effect on the tunnel excavation supporting.

(3) After the radial hole grouting consolidation, clear tunnel surface collapse of rock and soil, top-down slope excavation in a certain gradient. Because of all tunnels in the soft soil layer, the excavation, in the mouth of the cave, artificial may match machinery division excavation, dividing into blocks of the hole on the part, reducing the disturbance of soil. In rock area, we can consider to adopt two kinds of schemes for excavation: drilling and filling expanding agent on rock excavation and broken, forced to crack way; when comparing broken rock, artificial pick with a crusher for excavation. If demolition explosives must be used, 
we should reduce the dosage of blasting construction to the strong disturbance on around the surrounding rock, to ensure the stability of tunnel structure and safety.

(4) Tunnel temporary side slope of new excavation is used common anchor rod framework grass to anchor supporting in time.

Treatment Measures and Suggestions for the Tunnel Primary Retaining and Protecting Steel Frames, Steel Crossings Corroding

Combing with the quality test data in the expressway tunnel construction, the tunnel primary support steel frames, steel crossings corrosion probability is small. So the steel frames section, tunnel section room is more than $10 \mathrm{~cm}$ and complete excavation of the tunnel section, spraying $10 \mathrm{~cm} \mathrm{C25} \mathrm{concrete,} \mathrm{hanging}$ the diameter of $8 \mathrm{~mm}$ at $20 \times 20 \mathrm{~cm}$ steel meshes, each cross steel spacing add 6 roots reinforcement bolt. In steel frames section, the tunnel section room is more than $15 \mathrm{~cm}$ unfinished section down the steps of the tunnel excavation area, spraying $10 \mathrm{~cm} \mathrm{C} 25$ concrete, hanging the diameter of $8 \mathrm{~mm}$ at $20 \times 20 \mathrm{~cm}$ steel meshes, each cross steel spacing add 6 roots reinforcement bolt. In steel frames section, the tunnel section room between $5 \mathrm{~cm}$ and $10 \mathrm{~cm}$ and completed excavation area of the whole tunnel profile, spraying $10 \mathrm{~cm}$ C25 concrete, hanging the diameter of $8 \mathrm{~mm}$ at $20 \times 20 \mathrm{~cm}$ steel meshes, each cross steel add 8 roots reinforcement bolt. In a steel frame section, between $5 \mathrm{~cm}$ and $10 \mathrm{~cm}$ reserved space and the unfinished area of the whole tunnel section, down the steps, spraying $3 \mathrm{~cm} \mathrm{C} 25$ concrete, each cross steel anchor spacing and 8 roots reinforcement bolt.

\section{Treatment Measures and Suggestions for Cracks of Tunnel Gunite Concrete}

Tunnel crack width range between $0.12 \mathrm{~mm}$ and $0.12 \mathrm{~mm}$, the maximum crack width of $2.40 \mathrm{~mm}$, arch ring basic shape without large deformation, we use the net spray concrete reinforcement, before the reinforcement construction with high pressure water washing loose piece, moss, mud and other debris on the initial supporting surface.

\section{Treatment Measures and Suggestions for the Ins ufficient Thickness of Gunite Concrete}

The measured average thickness of shotcrete is less than the design thickness of tunnel, reinforcing primary support with net spray concrete. We use high pressure water washing loose piece, moss, mud and other debris on initial supporting surface, before the reinforcement construction.

Treatment Measures and Suggestions for Tunnel Lining Cavity

After testing, tunnel local paragraphs appear lining cavity, influencing supporting effect, and we need to carry out the radial grouting treatment to this disease, filling space, enhancing the supporting effect.

\section{Treatment Measures and Suggestions for Percolating Water of Tunnel Lining}

More obvious places of leakage of the tunnel lining, we use some ways to get a drain on emissions of drilling in a larger water seepage location, alleviating the static water pressure on lining damage, and connect the late implementation of tunnel longitudinal drainage pipeline with the drain pipeline.

\section{Treatment Measures and Suggestions for Karst Cave Collapse of Tunnel Hance}

At first, we use the pre-reinforcement processing, advanced ductule oblique grouting reinforcing karst caves, and in the cave mouth parts setting on a bed of the diameter of $22 \mathrm{~mm}$ at $20 \times 20 \mathrm{~cm}$ steel meshes, building $\mathrm{C} 15$ concrete, hanging steel fabric, add 3 meter-long the diameter of $25 \mathrm{~mm}$ hollow grouting reinforcing bolt, erecting I-shaped cross section NO.20B beams. At the same time embedded drainpipe end with the geotextile, and finally to reinforcing supporting.

\section{Treatment Measures and Suggestions for Ins ufficient Headspace of Tunnel Outline}

Preference of surrounding area: expanding the excavation section, spraying $10 \mathrm{~cm} \mathrm{C25}$ shotcrete, hanging the diameter of $8 \mathrm{~mm} 20 \times 20 \mathrm{~cm}$ steel fabric, and increasing 3 meter-long the diameter of $22 \mathrm{~mm}$ the system mortar anchor rod. Deviation of surrounding area: the whole section piecewise line by cut displacement of the cross beams, to expand section, adopt I shape steel frame or grille supporting the space, spraying C25 shotcrete, steel fabric, reserving $5 \mathrm{~cm}$ deformation. 


\section{CONCLUSIONS}

This article systematically summarized the tunnel continual construction after perennial work stoppage in three aspects: the disease types and the causes of disease and disease treatment measures and suggestions, consummating the existing engineering disease research of resume work construction project. The case discussed in this paper shows that comprehensive quality tests for established project, analysis of quality defects or diseases of all kinds of structures, and analyzing its formation mechanism, deep recognition of the influence factors of various kinds of structure component quality defect or disease severity, the classification and grading of recommendations of quality defect or disease, we provide some references for subsequent expressway reconstruction project program management, the design or construction of the expressway. It is effective to guide the reconstruction project completion inspection and acceptance, improving the efficiency of the expressway to return to work construction project investment. It also has important theoretical significance and application value.

\section{ACKNOWLEDGEMENTS}

First of all, I would like to acknowledge and extend my heartfelt gratitude to my supervisor-Associate Professor Xue Kaixi, for his vital encouragement and patient guidance, generous assistance and invaluable advice, all of which have been of inestimable worth to the completion of my thesis.

Secondly, my special thanks go to all people have helped and taught me in East China Institute of Technology.

Finally, I would also like to thank the support of the National Natural Science Foundation of China (project number: 41462011), College students' innovative training program in 2014 (project number: 2310400149) and Science and technology plan projects in Jiangxi province for 2012 (project number: 20123BBG70214).

\section{REFERENCES}

[1] Chen Zhongyue, Huang Miao, Road repair design resume work construction bridge disease, J. Journal of Anhui Architecture. 5 (2011) 141-142,144.

[2] Liu Houjian, Liu Zhiwei, Yan Zhihua, Northern shaanxi artificial high slope deformation and failure mode analysis and management of a certain power plant, J. Journal of Wuhan University: Engineering Science. 40 (2007) 566-569.

[3] Ma Laiqiu, Rong Zengxiang, Liao Hongbo, Tunnel slope failure mechanism study for Shi-man expressway, J. Journal of Transportation Science and Technology. 1 (2012) 42-44.

[4] Hong Naifeng, Steel corrosion in concrete and protection technology (2) - protection of reinforced concrete and steel corrosion electrochemical properties, J. Industrial Construction. 29 (1999) 58-61.

[5] Z.P. Bazant, Physical model for steel corrosion in concrete sea structures-theory, J. Journal of the Structural Division. 105 (1979) 1137-1142.

[6] Wu Ming, Steel corrosion mechanism and corrosion rate in concrete and practical prediction model research, D. Nanning: Guangxi University. 2013

[7] Luo Xin, Li Zhibin, Zheng Jiayan, The study of the expressway tunnel lining cavity behind, J. Modern Tunnel Technology. Supplement (2006) 79-82.

[8] Liu Huiying, Expressway tunnel disease formation mechanism and control measures research, D. Chengdu: Southwest Jiaotong University. 2007 